\title{
Timely HAART initiation may pave the way for a better viral control
}

\author{
Paola Paci ${ }^{1 *+}$, Federico Martini ${ }^{2}$, Massimo Bernaschi ${ }^{3}$, Gianpiero D'Offizi ${ }^{2}$, Filippo Castiglione ${ }^{3^{*}+}$
}

\begin{abstract}
Background: When to initiate antiretroviral therapy in HIV infected patients is a diffcult clinical decision. Actually, it is still a matter of discussion whether early highly active antiretroviral therapy (HAART) during primary HIV infection may influence the dynamics of the viral rebound, in case of therapy interruption, and overall the main disease course.

Methods: In this article we use a computational model and clinical data to identify the role of HAART timing on the residual capability to control HIV rebound after treatment suspension. Analyses of clinical data from three groups of patients initiating HAART respectively before seroconversion (very early), during the acute phase (early) and in the chronic phase (late), evidence differences arising from the very early events of the viral infection.

Results: The computational model allows a fine grain assessment of the impact of HAART timing on the disease outcome, from acute to chronic HIV-1 infection. Both patients' data and computer simulations reveal that HAART timing may indeed affect the HIV control capability after treatment discontinuation. In particular, we find a median time to viral rebound that is significantly longer in very early than in late patients.

Conclusions: A timing threshold is identified, corresponding to approximately three weeks post-infection, after which the capability to control HIV replication is lost. Conversely, HAART initiation occurring within three weeks from the infection could allow to preserve a significant control capability. This time could be related to the global triggering of uncontrolled immune activation, affecting residual immune competence preservation and HIV reservoir establishment.
\end{abstract}

\section{Background}

The question of when antiretroviral therapy has to be initiated remains a challenging issue. Recent studies show that the early immune response to HIV-1 infection is likely to be an important factor in determining the clinical course of disease [1]. The first weeks following HIV-1 transmission are extremely dynamic. They are associated with rapid damage to generative immune cell micro-environments and with immune responses that partially control the virus. Following HIV-1 infection, the virus first replicates locally in the mucosa and then is transported to draining lymph nodes where further amplification occurs. This initial phase of infection, until

\footnotetext{
*Correspondence: p.paci@iac.cnr.it; f.castiglione@iac.cnr.it

+ Contributed equally

${ }^{1}$ Biomedical University Campus, via Alvaro del Portillo 21, 00128 - Rome, Italy ${ }^{3}$ Institute for Computing Applications "Mauro Picone", National Research

Council, 00185 Rome, Italy

Full list of author information is available at the end of the article
}

the systemic viral dissemination begins, constitutes the eclipse phase [1]. In general, there is an exponential increase in plasma viremia with a peak 21-28 days after infection. By this time, significant depletion of mucosal $\mathrm{CD} 4^{+} \mathrm{T}$ cells has already occurred. Around the time of peak viremia, patients may become symptomatic and reservoirs of latent virus are established $[1,2]$.

The "window of opportunity" between the infection and peaking of viremia, prior to massive $\mathrm{CD} 4^{+} \mathrm{T}$ cell destruction and the establishment of viral reservoirs, seems to be a narrow but crucial period in which an antiretroviral therapy can control viral replication, prevent an extensive $\mathrm{CD} 4^{+} \mathrm{T}$ cell depletion from occurring and curb generalized immune activation. Thus, thwarting HIV replication by introducing HAART in the early phases of infection could have a substantial impact on the whole disease course. In particular, suggested factors that may contribute to the observed better viral control after treatment interruption in very early treated

\section{Biomed Central}


patients are [3]: $i$ ) early arrest of viral escape, leaving the virus vulnerable; $i$ ) preservation or even enhancement of the immune response resulting from the early clearing of antigen; iii) prevention of the establishment of a pool of HIV-specific memory CD4 T cells thus leaving fewer target cells available for viral infection.

An ideal clinical model aimed at addressing such issue should compare a number of patients treated starting on different times: from very early to very late. Besides the ethical issues, it is rather difficult to collect enough patients to significantly represent the whole spectrum of possible HAART initiation timings. As a matter of fact, a practical clinical model would compare very early to late-treated patients. While informative on the overall role of HAART timing on disease course, this approach would not allow to verify if there are events in the early infection influenced by the starting time of HAART that affect directly and decisively the course of the disease.

We have already shown in [4-6] that an agent-based model of HIV-1 infection could be a valuable tool for the study of the AIDS disease progression and treatment. The computerized simulation allows us to track the effect of HAART timing on the progression of the disease.

The aim of the present work is to verify the effect of HAART timing on subsequent events. Indeed, both a clinical model and a computational simulation show that a late initiation of treatment affects $\mathrm{HIV}-1$ replication control. Interestingly, the in silico model identifies a significant three-week time threshold as the "ultimate" time point beyond which the decisive HIV-induced damages already occurred, affecting the whole disease course.

In a previous work [4], we analyzed clinical data of patients initiating HAART within six months from infection (i.e., we called that early phase) and performed computer simulations to predict the differences in viral rebound at therapy interruption between those patients and subjects initiating therapy during the chronic phase (i.e., we called that late phase, corresponding to initiating six or more months after infection). Our conclusion was that early initiation of therapy does not prolong the disease-free period when compared to a treatment started during the late phase. However, other studies suggest that an earlier initiation is preferable [7-9]. This motivated us to better identify the meaning of early initiation. In the present article, we extend the analysis in [4] to get a more complete picture. We analyze clinical data of very early patients (i.e., treated before seroconvertion) against late-treated patients.

\section{Methods}

\section{Clinical studies}

We analyzed the results of clinical studies performed at the Clinical Department of the National Institute for Infectious Disease "L. Spallanzani” in Rome.
A first group of eleven patients ( 9 male and 2 female) were diagnosed HIV-1 positive between year 1998 and 2006. All patients initiated HAART within 14 days from diagnosis, during the very early phase of infection (see Table 1). The very early phase was defined as having a negative or indeterminate western blot for HIV-1 antibodies in combination with a positive test for either p24 antigen or a detectable HIV-1 RNA concentration. Those patients were treated with zidovudine/lamivudine (CBV) in combination with either the reverse transcriptase inhibitor efavirenz (EFZ) or one protease inhibitor lopinavir/ritonavir (KAL) or indinavir (IDV). Because anaemia and neutropenia were diagnosed, in two cases CBV has been substituted with lamivudine (3TC) and staduvine (D4T). All those patients underwent a therapy cycle for $2 \pm 1$ years and remained off HAART for about 48 weeks.

The second group is made up by twenty-two patients (21 male and 1 female) enrolled in the program between year 1998 and 2005. Patients in this group underwent HAART during the early phase of HIV-1 infection. In particular, they started HAART about 20 days after treatment diagnosis (see Table 2). Early patients were defined as having documented seronegative HIV-1 antibody test within the previous 6 months; acute symptomatic seroconversion illness; evolving HIV-specific antibody response by ELISA; positive HIV-DNA PCR in PBMC. Those patients were treated with three different drugs (in the majority of cases zidovudine (AZT) plus $3 \mathrm{TC}$ plus a protease inhibitor. Further details can be found in Table One of [4]. All those patients underwent a therapy cycle for $3 \pm 1$ years and remained o HAART for about 88 weeks.

The third group consists of twenty-one patients (12 male, 9 female). They started HAART during the chronic phase of infection defined as suggested by the guidelines [10]. In particular, they started HAART about 3.5 years after treatment diagnosis (see Table 3 ). Their CD4 count at initiation was $400 \pm 150$ per microlitre of plasma. The range of calendar year for starting HAART among those patients was $1998 \pm 3$. All those patients underwent a therapy cycle for $4 \pm 2$ years and remained off HAART for about 41 weeks. The Ethical Committee of the "L. Spallanzani" Institute approved the study and the patients gave a written informed consent.

\section{Plasma HIV-1 determination}

Plasma HIV-1 RNA levels were determined by a secondgeneration assay based on nucleic acid sequence based amplification (NASBA), for samples collected until 2001 and by the branched-chain DNA assay (Versant HIV RNA test, Version 3.0, lower limit of quantification 50 copies/ml; Bayer Diagnostics, Milan, Italy) from 2001 until 2008. 
Table 1 Very early subjects with an immediate treatment before seroconversion

\begin{tabular}{|c|c|c|c|c|c|c|c|c|}
\hline \multirow[t]{2}{*}{ ID } & \multirow[t]{2}{*}{ Therapy $^{\dagger}$ therapy } & \multirow[t]{2}{*}{ Elapsed days ${ }^{\ddagger}$ therapy } & \multicolumn{2}{|c|}{ At diagnosis* } & \multicolumn{2}{|c|}{4 weeks after** } & \multicolumn{2}{|c|}{8 weeks after** } \\
\hline & & & CD4 & vRNA & CD4 & vRNA & CD4 & vRNA \\
\hline Pz4 & CBV, IDV & 14 & 603 & 16000 & 800 & $<50$ & 792 & 6000 \\
\hline Pz34 & CBV, EFZ & 18 & 370 & 280000 & 647 & 640 & 815 & $<50$ \\
\hline Pz36 & CBV, EFZ & 15 & 402 & 14000 & & $<50$ & 599 & $<50$ \\
\hline Pz38 & CBV, EFZ & 2 & 543 & 3500000 & & 1600 & 770 & 220 \\
\hline Pz41 & CBV, EFZ & 12 & 568 & 1300000 & 1549 & $<50$ & 857 & $<50$ \\
\hline Pz42 & CBV, EFZ & 8 & 234 & & 1083 & 2300 & 657 & $<50$ \\
\hline Pz47 & CBV, IDV & 4 & 753 & 8802 & 615 & 220 & 743 & $<50$ \\
\hline Pz51 & CBV, EFZ & 10 & 530 & 91972 & 655 & $<50$ & 810 & $<50$ \\
\hline Pz67 & CBV, KAL & 0 & 797 & 21675 & 884 & 98 & 932 & $<50$ \\
\hline Pz83 & CBV, KAL & 18 & 426 & $>500000$ & 770 & 2349 & 1524 & 1037 \\
\hline Pz113 & CBV, KAL & 3 & 261 & 65716 & 422 & 660 & 460 & 302 \\
\hline
\end{tabular}

Clinical information about the eleven patients selected at the Clinical Department of the National Institute for Infectious Disease "L. Spallanzani" in Rome. All subjects received HAART within six months from primary infection.

${ }^{+}$CBV, Combivir (AZT Zidovudine plus 3TC Lamivudine); IDV, Indinavir; EFZ, Efavirenz; KAL, Kaletra (lopinavir/ritonavir). ${ }^{\ddagger}$ Days elapsed from diagnosis (enrollment) to initiation of HAART. ${ }^{*} \mathrm{CD} 4$ and CD8 are per microlitre of plasma, viremia is per millilitre of plasma. ${ }^{*} 4$ weeks after treatment interruption.

\section{Computational model}

The current version of the model we employ derives from an early simulator that has been quite extensively described in previous publications [11,12]. Recently it has been specialized to simulate the HIV-1 infection [6] and the effects of antiretroviral therapy [4].
Briefly, it resorts to bit strings to represent "binding sites" of cells and molecules, as for example lymphocyte receptors, MHC, antigen peptides and epitopes, immunocomplexes, etc. [13]. The model includes the major classes of cells of the lymphoid lineage ( $\mathrm{T}$ helper lymphocytes, cytotoxic $\mathrm{T}$ lymphocytes, B lymphocytes and

Table 2 Early subjects with an immediate treatment of acute HIV-1 infection

\begin{tabular}{|c|c|c|c|c|c|c|c|c|c|c|}
\hline \multirow[t]{2}{*}{ ID } & \multirow[t]{2}{*}{ Therapy $^{\dagger}$} & \multirow[t]{2}{*}{ Elapsed days ${ }^{\ddagger}$} & \multirow[t]{2}{*}{ Days on therapy } & \multirow[t]{2}{*}{ Days out therapy } & \multicolumn{3}{|c|}{ At diagnosis* } & \multicolumn{3}{|c|}{ At first interruption } \\
\hline & & & & & CD4 & CD8 & vRNA & CD4 & CD8 & vRNA \\
\hline Pt 03 & D4T,3TC,IDV & 14 & 1775 & 27 & 653 & 1659 & 75 & 809 & 459 & 5794 \\
\hline Pt 05 & AZT,3TC,IDV & 61 & 1657 & 27 & 444 & 633 & 100 & 867 & 476 & $<50$ \\
\hline Pt 29 & AZT,3TC,NFV & 14 & 1069 & 28 & 1103 & 1964 & 31 & 741 & 844 & $<50$ \\
\hline Pt 33 & AZT,3TC,IDV & 66 & 925 & 28 & 424 & 991 & 0.13 & 458 & 474 & 147 \\
\hline Pt 35 & AZT,3TC,EFV & 1 & 1041 & 30 & 522 & 1205 & 78 & 919 & 607 & 496 \\
\hline Pt 37 & AZT,3TC,EFV & 0 & 1371 & 790 & 545 & 1233 & 3.9 & 901 & 384 & $<50$ \\
\hline Pt 58 & AZT,3TC,EFV & 3 & 442 & 27 & 768 & 1632 & 50 & 1451 & 1580 & $<50$ \\
\hline Pt 06 & AZT,3TC,IDV & 32 & 1620 & 1908 & 882 & 4146 & 4.1 & 754 & 726 & $<50$ \\
\hline Pt 18 & AZT,3TC,IDV & 26 & 635 & 213 & 1319 & 1810 & 15 & 1718 & 825 & $<80$ \\
\hline Pt 24 & AZT,3TC,IDV & 3 & 1231 & 542 & 507 & 3162 & 78 & 513 & 1067 & $<50$ \\
\hline Pt 31 & AZT,ЗTC,EFV & 19 & 1102 & 1654 & 322 & 243 & 490 & 1154 & 1165 & $<50$ \\
\hline Pt 41 & AZT,3TC,EFV & 12 & 705 & 1837 & 568 & 278 & 130 & 1326 & 940 & $<50$ \\
\hline Pt 45 & AZT,3TC,EFV & 5 & 488 & 55 & 307 & 1032 & 130 & 872 & 506 & $<50$ \\
\hline Pt 53 & AZT,3TC,Lop/rit & 16 & 503 & 179 & 341 & 779 & 18.9 & 545 & 529 & 150 \\
\hline Pt 04 & AZT,3TC,IDV & 6 & 1561 & 2155 & 603 & 1288 & 1.6 & 1047 & 1015 & 88 \\
\hline Pt 19 & AZT,3TC,IDV & 13 & 1480 & 1640 & 1338 & 716 & 10 & 1113 & 447 & $<50$ \\
\hline Pt 28 & AZT,3TC,NFV & 7 & 325 & 70 & 281 & 860 & 190 & 863 & 485 & $<80$ \\
\hline Pt 32 & AZT,3TC,EFV & 15 & 1384 & 669 & 409 & 1448 & 4.3 & 960 & 331 & $<50$ \\
\hline Pt 72 & AZT,3TC,LPV & 7 & 318 & 343 & 827 & 386 & 19 & 1005 & 751 & $<50$ \\
\hline Pt 81 & AZT,3TC,NVP & 33 & 745 & 317 & 412 & 264 & 32.5 & 522 & 461 & 68 \\
\hline Pt 85 & AZT,3TC,Lop/rit & 46 & 717 & 717 & 326 & 669 & 50 & 855 & 1436 & $<50$ \\
\hline Pt 92 & 3TC,Lop/rit,TNF & 0 & 455 & 331 & 616 & 3774 & 46.3 & 1027 & 913 & $<50$ \\
\hline
\end{tabular}

Clinical information about the twenty-two patients selected at the Clinical Department of the National Institute for Infectious Disease "L. Spallanzani" in Rome. All subjects received HAART within six months from primary infection.

${ }^{\dagger}$ D4T, staduvine; 3TC, lamivudine; IDV, Indinavir; AZT, Zidovudine; NFV, nelflnavir; EFV, Efavirenz; NVP, nevirapine; TNF, Tenofovir; Lop, lopinavir; Rit, Ritonavir; LPV $(\mathrm{KAL})$, Kaletra (lopinavir/ritonavir). ${ }^{\ddagger}$ Days elapsed from diagnosis (enrollment) to initiation of HAART. * ${ }^{*} \mathrm{CD}$ and CD8 are per microlitre of plasma, viremia is per millilitre of plasma. 
Table 3 Late subjects with deferred treatment of acute HIV-1 infection

\begin{tabular}{|c|c|c|c|c|c|c|c|c|c|c|}
\hline \multirow[t]{2}{*}{ ID } & \multirow[t]{2}{*}{ Therapy $^{\dagger}$} & \multirow[t]{2}{*}{ Elapsed days ${ }^{\ddagger}$} & \multirow[t]{2}{*}{ Days on therapy } & \multirow[t]{2}{*}{ Days out therapy } & \multicolumn{3}{|c|}{ At diagnosis* } & \multicolumn{3}{|c|}{ At first interruption } \\
\hline & & & & & CD4 & CD8 & vRNA & CD4 & CD8 & vRNA \\
\hline Pt 07 & AZT, 3TC & 300 & 1303 & 606 & 463 & 2498 & 34000 & 614 & 3101 & 9154 \\
\hline Pt 12 & D4T, 3TC, SQV & 26 & 1117 & 89 & 422 & 754 & 62000 & 601 & 948 & $<50$ \\
\hline Pt 16 & $A Z T, d d C$, Rit & 9 & 1803 & 66 & 116 & 1170 & 990000 & 483 & 1165 & $<50$ \\
\hline Pt 17 & AZT, 3TC & 49 & 1872 & 412 & 51 & 1155 & 110000 & 693 & 1359 & 150 \\
\hline Pt 18 & $A Z T, d d l$ & 268 & 1615 & 558 & 340 & 994 & 35000 & 989 & 1172 & $<50$ \\
\hline Pt 19 & AZT, 3TC & 393 & 819 & 206 & 559 & 1230 & 70319 & 616 & 825 & $<50$ \\
\hline Pt 20 & AZT, 3TC & 15 & 2503 & 21 & 235 & 877 & 230000 & 566 & 1246 & $<50$ \\
\hline Pt 22 & D4T, 3TC & 0 & 1807 & 64 & 290 & & 140000 & 1334 & & $<50$ \\
\hline Pt 23 & $A Z T, 3 T C$ & 483 & 2109 & 248 & 409 & 734 & 47000 & 686 & 572 & $<50$ \\
\hline Pt 25 & D4T,3TC, IDV & 22 & 1661 & 625 & 383 & 809 & 37000 & 896 & 1208 & $<50$ \\
\hline Pt 26 & AZT, 3TC & 38 & 841 & 1150 & 540 & 1256 & 16000 & 719 & 640 & $<50$ \\
\hline Pt 27 & AZT,3TC & 10 & 1885 & 50 & 332 & & 2800 & 636 & & $<50$ \\
\hline Pt 28 & AZT,3TC,EFV & 0 & 3131 & 373 & 556 & & 1900 & 752 & & $<50$ \\
\hline Pt 29 & AZT,3TC,Lop/rit & 21 & 1950 & 69 & 483 & & 150000 & 1233 & & 100 \\
\hline Pt 31 & AZT,3TC,IDV & 0 & 1255 & 109 & 574 & 1625 & 7900 & 813 & 640 & $<50$ \\
\hline Pt 32 & AZT,3TC,IDV & 0 & 1472 & 160 & 393 & & 1335 & 1235 & & $<50$ \\
\hline Pt 33 & D4T, 3TC & 407 & 523 & 66 & 479 & 494 & 473 & 705 & 450 & $<50$ \\
\hline Pt 34 & ddl, D4T, NVP & 13 & 2207 & 54 & 493 & 1628 & 230000 & 907 & 943 & $<50$ \\
\hline Pt 38 & AZT,3TC,LPV & 0 & 1460 & 270 & 481 & 1156 & 20000 & 534 & 964 & $<50$ \\
\hline Pt 39 & AZT,3TC,NVP & 64 & 2734 & 767 & 539 & & 41000 & 859 & & $<50$ \\
\hline Pt 41 & AZT,3TC,Lop/rit & 62 & 428 & 89 & 200 & & 135000 & 848 & & $<50$ \\
\hline
\end{tabular}

Clinical information about the twenty-one patients selected at the Clinical Department of the National Institute for Infectious Disease "L. Spallanzani" in Rome. All subjects received HAART during chronic phase of infection.

${ }^{\dagger}$ AZT, Retrovir (Zidovudine); 3TC, Epivir (Lamivudine); D4T, Zerit (Staduvine); SQV, Saquinvir (Invirase); Zalcitabina (ddC, Hivid); Rit, Ritonavir (Norvir); ddl, Didanosine (Videx); IDV, Indinavir (Crix-ivan); EFV, Efavirenz; NVP, Nevirapine (Viramune); LPV (Lop), Lopinavir; ${ }^{\ddagger}$ Days elapsed from diagnosis (enrollment) to initiation of HAART. ${ }^{*} \mathrm{CD} 4$ and $\mathrm{CD} 8$ are per microlitre of plasma, viremia is per millilitre of plasma.

antibody-producer plasma cells) and some of the myeloid lineage (macrophages and dendritic cells). These entities are individually represented. In contrast to cells, cytokines like interleukin-2 are represented in terms of concentrations and their dynamics described by a parabolic partial differential equation plus a degradation term accounting for the finite half-life [5,14]. Modeling features of the HIV infection include HIV replication inside infected lymphocytes, $\mathrm{T}$ production impairment; specific response against HIV strains and HIV mutation.

The simulated life cycle of the virus is represented by the following stages: 1) the virus infects $\mathrm{CD} 4^{+} \mathrm{T}$ cells, macrophages, dendritic cells; 2) reverse transcriptase copies the viral single stranded RNA genome into a double-stranded viral DNA. The viral DNA is then integrated into the host chromosomal DNA; 3) the virus remains at rest until an event activates the transcription; 4) the replicating virus buds from the cell membrane. Fully assembled virions are then able to infect other cells to restart the life cycle. HAAR effects are modeled as follows: Reverse transcriptase inhibitors block reverse transcriptase enzymatic functions and avoid completion of synthesis of the double-stranded viral DNA thus preventing HIV-1 from replicating (i.e., it prevents the virus in stage 1 from reaching stage 2); Protease inhibitors prevent viral replication by inhibiting the activity of HIV-1 protease, an enzyme used by the virus to cleave nascent proteins for final assembly of new virions (i.e., it prevents virus assembly in stage 4). Further details and parameter settings of the simulations can be found in the Additional file 1 .

For what concerns the setting of the parameters related to the therapy, we performed computer simulations in which we fixed the immunological parameters at the time of therapy initiation on the basis of the average values measured in patients in vivo: $5.8 \pm 0.2$ RNA copies/ $m l$ (in logarithmic scale), $870 \pm 50 \mathrm{CD} 4$ cells $/ \mu l$ and $430 \pm 50 \mathrm{CD} 8$ cells/ $\mu l$. For all simulations we applied a one-year course of HAART. Further details on the tuning of the simulation parameters can be found in the Additional file 2.

\section{Results}

We analyze virological data from HIV patients treated during the very early, early and late phase of infection and compare them with computer simulations.

In Figure 1 clinical data of all three analyzed groups is shown altogether. In a point-by-point comparison we 


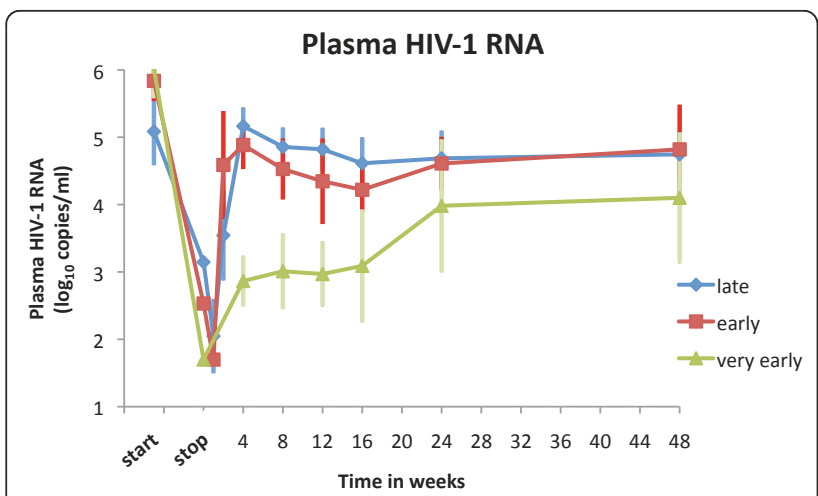

Figure 1 Plasma HIV-1 RNA load. The mean plasma HIV-1 RNA load versus time in weeks after interruption of HAART for patients classified in late (21 subjects), early (22 subjects) and very early (11 subjects) groups. Error bars show standard deviations. The features of the three clinical settings are given in section Patients and Methods. find no statistical difference in viral rebound between early and late-treated patients $(\mathrm{P} \geq 0.05$, Mann-Whitney $\mathrm{U}$ two-tailed test) confirming the results of [4]. In addition, we observe that a difference does exist for very early initiation of therapy $(\mathrm{P}<0.05$, Mann-Whitney $\mathrm{U}$ two-tailed test).

In the present work we extend the simulations of [4] to include the new clinical settings corresponding to a very early initiation of therapy. In particular, the very early simulation settings correspond to a beginning of the therapy within the first week whereas the late settings correspond to initiating therapy between week five and six from infection.

Figure 2 summarizes data of virological rebound (averages) after therapy interruption at different time points (4, 8 and 24 weeks) for very early and late patients for both clinical (empty boxes) and simulation data (filled

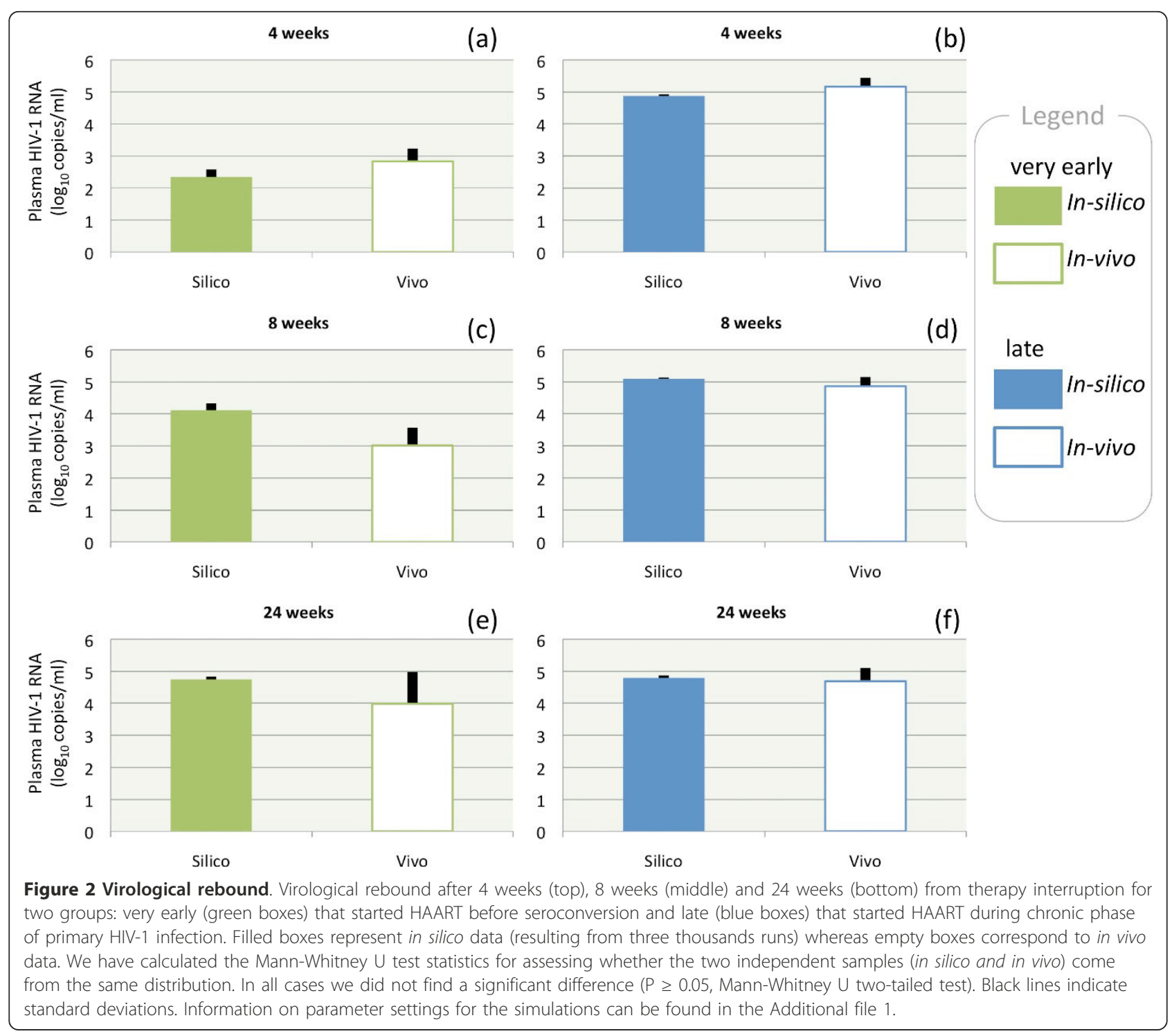


boxes). Firstly, the figure shows that clinical and simulation data are in good agreement (differences are not statistically significant: $\mathrm{P} \geq 0.05$, Mann-Whitney U two-tailed test). Secondly, the difference in plasma HIV-1 RNA (copies/ml) between very early and late-treated patients decreases with increasing time from therapy interruption. Panel (a) shows a difference of about two logs with panel (b) for both clinical data and simulations. These differences vanish after 24 weeks from therapy interruption (cfr. panel (e) and (f)). The overall message is that a delay in the initiation of therapy reduces the chances of maintaining a therapy effect at discontinuation.

In order to provide a more precise estimate of the time "limit" beyond which the benefit of an early initiation of therapy vanishes, we use the simulation to investigate the influence of HAART initiation time $\left(t_{s}\right)$ on the viral rebound. The corresponding results are shown in Figure 3. The virological rebound at one week after therapy interruption as a function of $t_{s}$ is presented. We observe that there are two regimens, one for $t_{s}<20$ days and one for $t_{s}>30$ corresponding to what clinicians call respectively best controllers (with undetectable HIV RNA levels) and rebounders (whose HIV viremia load returns, approximately, to the pre-HAART level).

The points in Figure 3 fit pretty well a generalized logistic function (i.e., the Richards' curve, [15]) describing the growth of viremia as a function of $t_{s}$,

$$
V\left(t_{s}\right)=a+\frac{k-a}{1+e^{-d\left(t_{s}-t_{s}^{*}\right)}}
$$

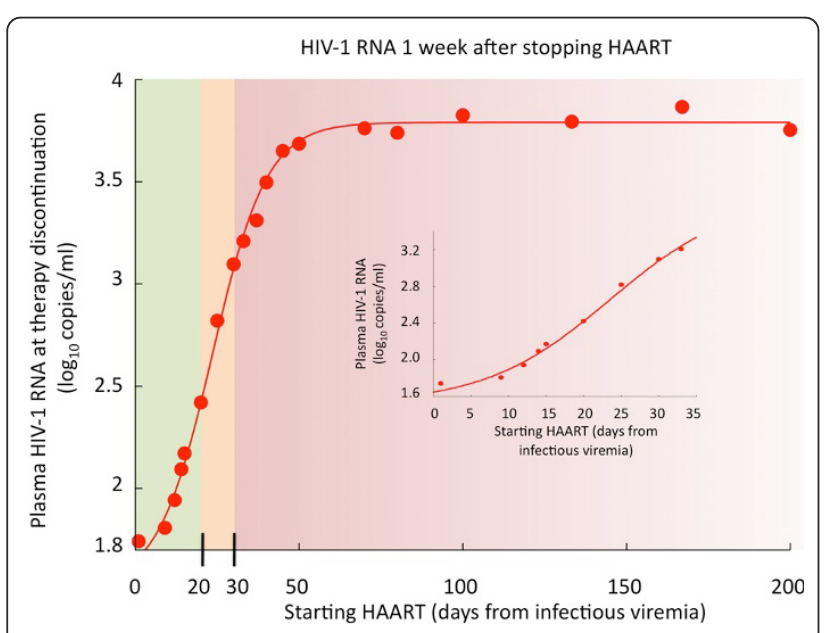

Figure 3 Virological rebound after 1 weeks. Virological rebound at 1 week after therapy interruption for different starting time points of HAART $\left(t_{s}\right)$. The red dots represent the results of thousands simulations and the fitting line is given by the Richards' curve in equation 1. Standard deviation is about $0.1 \log _{10}$ (copies $/ \mathrm{ml}$ ) for all points. Inset plot is a zoom for $t_{s}<30$ days. Parameters of the fitting curve are: $a=1.52, k=3.79, d=0.12$, and $t_{s}^{*}=23.50$. where the parameter $k$ is the carrying capacity or the upper asymptote, $a$ is the lower asymptote, $d$ is the growth rate, and $t_{s}^{*}$ is the time of maximum growth. By moving the time of the measurements beyond one week after therapy interruption, the resulting data still fit the same $V\left(t_{s}\right)$ but with a greater $a$, a smaller $\mathrm{d}$ and a greater $t_{s}^{*}$. In particular the limit for $\mathrm{d}$ going to zero, of $V\left(t_{s}\right)$ is $(a+k) / 2$ may lead to the deceiving conclusion that there is no window of opportunity because the viral rebound is independent from $t_{s}$.

With respect to $t_{s}^{*}$, the value of $\sim 23: 5$ days points to the early inflammation as a critical phase of the disease. To bring into focus this facet, we compare two simulated "markers" of the inflammation state in untreated (control case), very early and lately treated simulated patients (see Figure 4). These virtual markers are given by the cell counts of active macrophages (a) and dendritic cells presenting viral proteins on class II MHC molecules (b). We observe that the late-treated case is comparable to the control case (untreated) whereas the very early stands on its own. This observation suggests that it is the activation of the immune system through the set up of an in ammatory state that has to be blamed for the increased viral rebound for $t_{s}>t_{s}^{*}$.

Figure 4 shows with clarity that very early initiation of the treatment can down-regulate the immune activation, hence limiting viral replication and spread. Interestingly, this view is supported by the observation that HIV triggers the immune activation directly (e.g., HIV gene products can induce the activation of lymphocytes and macrophages as well as the production of pro-inflammatory cytokines and chemokines [2]) or indirectly (e.g., sustained antigen-mediated immune activation occurs in HIV-1-infected patients due also to other viruses like the cytomegalovirus or the Epstein-Barr virus [2]). In both case, the result is a high level of pro-inflammatory cytokines, such as tumor necrosis factor alpha, interleukin 6 and interleukin 1 beta, right from the early stages of HIV-1 infection [2].

\section{Discussion}

Recent analysis (performed by Fiebig et al. [16]) of samples from individuals that have been infected by HIV-1 has revealed that patients can be categorized into six stages on the basis of a sequential gain in positive HIV1 clinical diagnostic assays (viral RNA measured by PCR, p24 and p31 viral antigens measured by enzymelinked immunosorbent assay (ELISA), HIV-1-specific antibody detected by ELISA and HIV-1-specific antibodies detected by western blot, [16]). Patients progress from acute to early chronic infection at the end of stage 


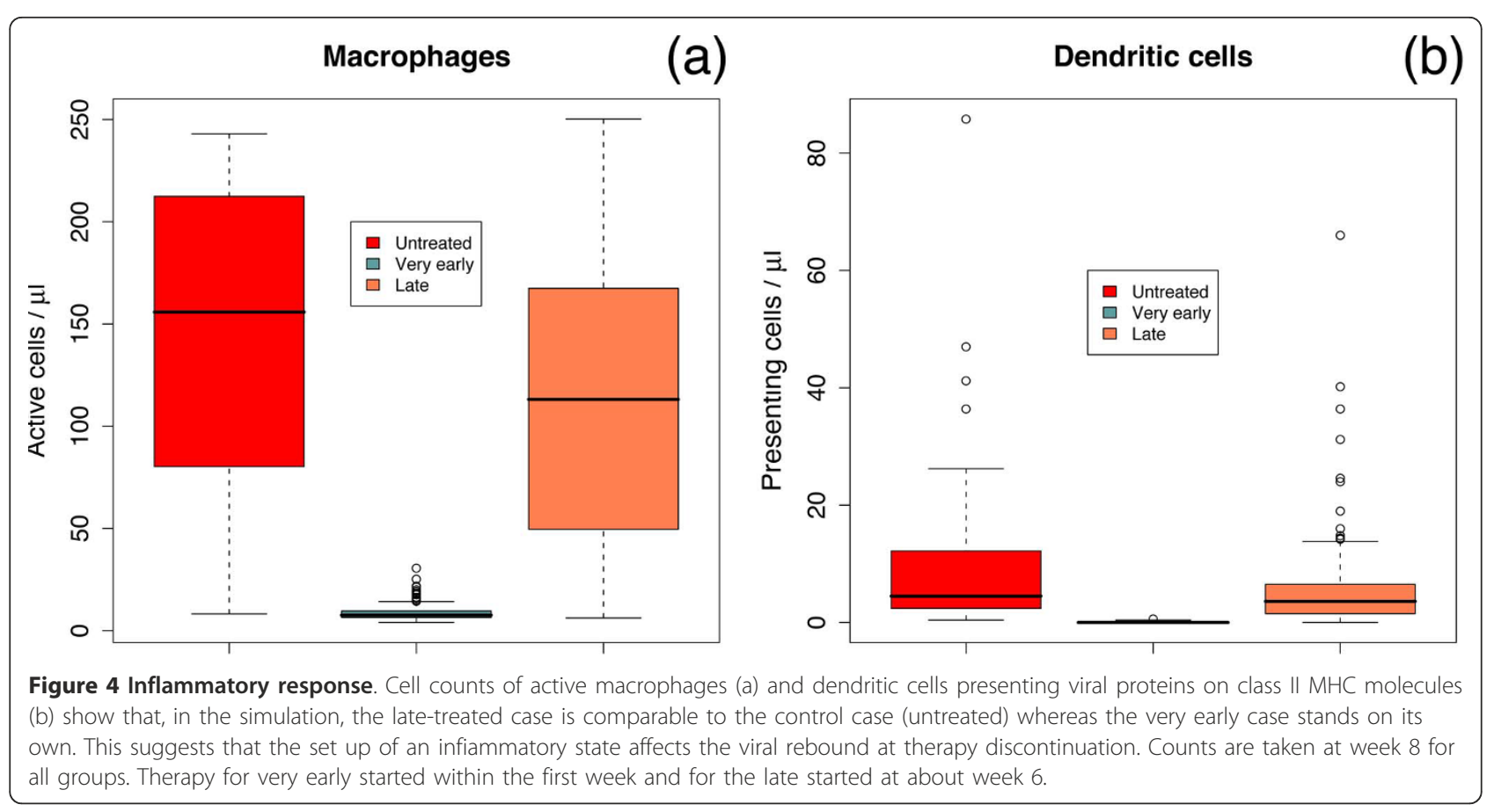

$\mathrm{V}$, approximately 100 days following infection, as the plasma viral load begins to stabilize.

With respect to the study conducted by clinical data analysis and computer simulation described so far, we identify three regimens, as highlighted in Figure 3. These can be paralleled to Fiebig et al. stages [16]. In particular, we observe that patients treated with HAART in very early stages of the infection (stage I-III) are likely to better control the viremia after treatment interruption [3]. If therapy starts in the acute phase (stage V-VI) then the action of the drug foils the immune response and, as a consequence, at the end of the therapeutic period, the virus rebounds undisturbed.

These considerations are summarized in Figure 5 where we draw a schematic picture of the importance of an early initiation of HAART with respect to the progression of HIV markers according to Fiebig's et al. stages. In particular we identified the "window of opportunity" corresponding to stages I-III, that is, the first three weeks from primary HIV-1 infection. Patients receiving therapy in this narrow period are likely to turn out to be best controllers. Probably, the massive immune activation in the early stage of the disease favors the virus, as it finds more host target cells to exploit for replication. In point of fact, the ensuing massive depletion of CD4+ T cells in mucosal lymphoid tissues, can result in the disruption of the mucosal barrier in the gut. This barrier prevents the translocation of the intestinal flora from the gut to the systemic immune system restricting it to the lamina propia and the mesenteric lymph nodes [2]. HIV-1 infection is indeed associated with a significant increase of plasma lipopolysaccharide levels that is an indicator of microbial translocation, directly correlated with measures of immune activation.

\section{Conclusions}

A number of studies indicate that interfering with HIV replication by starting the therapy in the early phases of the infection could have a deep impact on the whole disease course. However, HAART is costly, it is onerous for both patient and health care provider, and often brings adverse effects. Its clinical benefit must therefore be weighed against its burden.

In the present study, we resorted to a computer model to study the dynamics of the plasma viral load after prolonged treatment interruption in two groups of in silico patients: those who initiate HAART very early and those who start it lately. We evaluated the model comparing the results to clinical data. We found that an opportunity time-window exists for the initiation of HAART (roughly within three weeks before the establishment of viral reservoirs), in which the therapy can control viral replication, preventing generalized immune activation and extensive CD4+ T cell depletion.

\section{Availability and requirements}

An educational version of the immune system simulator is available on our website:

- Name: C-ImmSim

- Home page: http://www.iac.rm.cnr.it/ filippo/CImmSim.html 


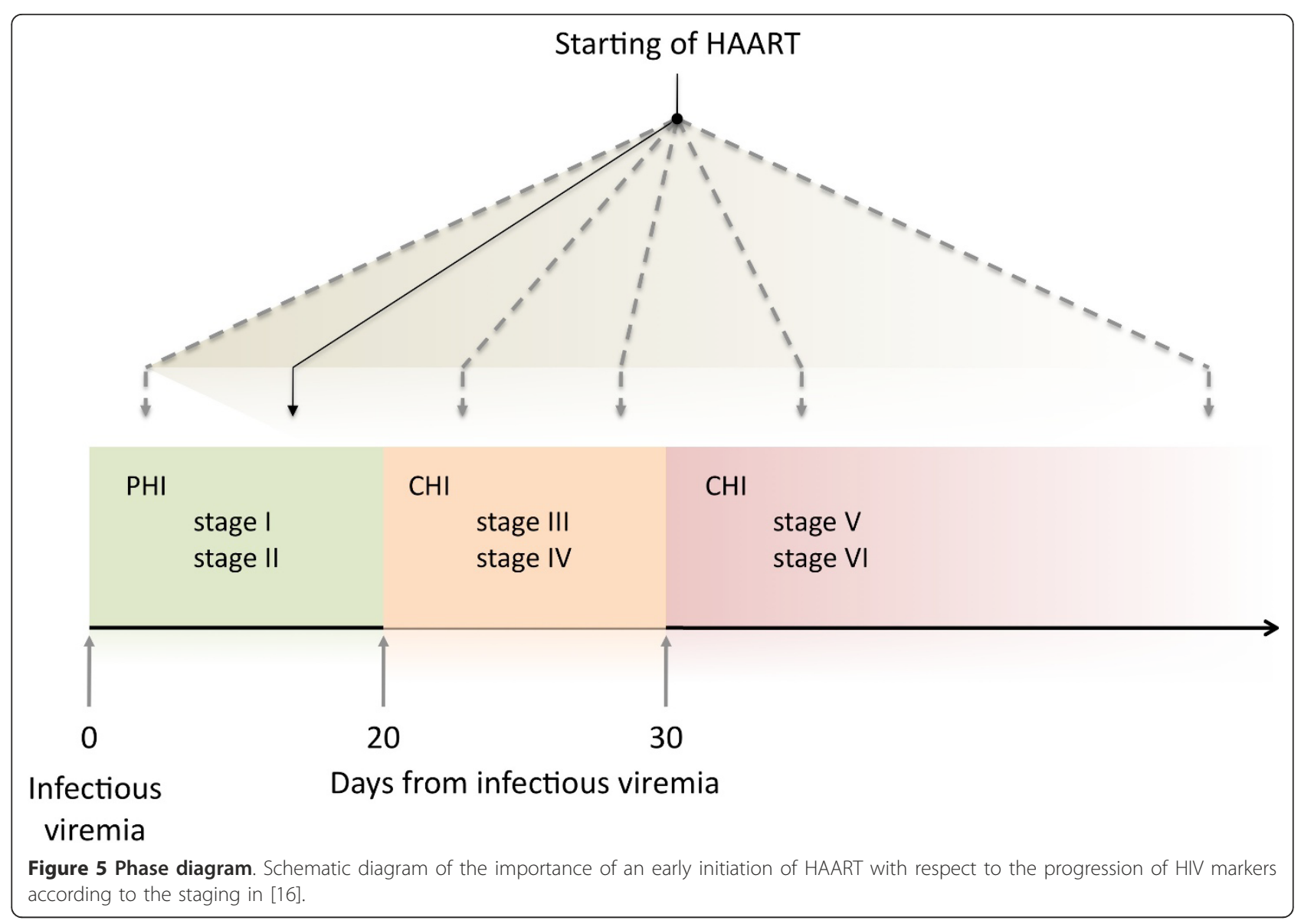

- Operating system(s): Linux, Unix Mac OS X, Windows

- Programming language: $\mathrm{C}$

- Licence: C-ImmSim is available under a LICENSE AGREEMENT that needs to be signed: http://www. iac.rm.cnr.it/ filippo/how-to-get-cimmsim_files/ LicenseAgreement.pdf

\section{Additional material}

Additional file 1: Mathematical model details. This file lists all the interactions between cells and molecules considered in the model and an accurate description of the parameter setting.

Additional file 2: Parameters tuning for the simulation of HIV infected virtual patients. This file reports details of the parameter setting and tuning.

\section{Acknowledgements}

We acknowledge Rossella Carello and Chrysoula Vlassi for assistance in data extraction. We wish to acknowledge support of the European Community through the contract FP6-2004-IST-4, No. 028069 (ImmunoGrid). We thank the "Consorzio interuniversitario per le Applicazioni di Supercalcolo Per Universitá e Ricerca" (CASPUR) for computing resources and support (Standard HPC grant 2009 and 2010).

\section{Author details}

${ }^{1}$ Biomedical University Campus, via Alvaro del Portillo 21, 00128 - Rome, Italy. ${ }^{2}$ National Institute for Infectious Diseases "Lazzaro Spallanzani", I.R.C.C.S., 00149 Rome, Italy. ${ }^{3}$ Institute for Computing Applications "Mauro Picone",

National Research Council, 00185 Rome, Italy.

\section{Authors' contributions}

FC and PP designed and performed research; all authors wrote the paper. FM and GDO provided the clinical data. All authors read and approved the final manuscript.

\section{Competing interests}

The authors declare that they have no competing interests.

Received: 25 October 2010 Accepted: 1 March 2011

Published: 1 March 2011

\section{References}

1. McMichael AJ, Borrow P, Tomara GD, Goonetilleke N, Haynes BF: The immune response during acute HIV-1 infection: clues for vaccine development. Nat Rev Immunol 2010, 10:11-23.

2. Appay $V$, Sauce $D$ : Immune activation and inflammation in HIV-1 infection: causes and consequences. J Pathol 2008, 214:231-241.

3. Steingrover R, Pogány K, Garcia EF, Jurriaans S, Brinkman K, et al: HIV-1 viral rebound dynamics after a single treatment interruption depends on time of initiation of highly active antiretroviral therapy. AIDS 2008, 22:1583-1588.

4. Paci P, Carello R, Bernaschi M, D'Offizi G, Castiglione F: Immune control of HIV-1 infection after therapy interruption: immediate versus deferred antiretroviral therapy. BMC Infect Dis 2009, 9:172. 
5. Paci P, Castiglione F, Bernaschi $m$, Baldazzi V: A discrete/continuous model of anti-HIV response and therapy. IEEE Computer Society, Digital library Proceedins UKSIM 2008, 481-486.

6. Castiglione F, Poccia F, D'Offizi G, Bernaschi M: Mutation, Fitness, Viral Diversity, and Predictive Markers of Disease Progression in a Camputational Model of HIV Type 1 infection. AIDS Res Hum Retrovirus 2004, 20(12):1314-1323.

7. Ahdien-Grant L, Yamashita TE, Phair JP, Detels R, Wolinsky SM, et al: When to Initiate Highly Active Antiretroviral Therapy: A Cohort Approach. Am J Epidemiol 2003, 157:738-746.

8. Autran B, Carcelain G, Li TS, Blanc C, Mathez D, et al: Positive Effects of Combined Antiretroviral Therapy on $\mathrm{CD}^{+}{ }^{+}$Cell Homeostasis and Function in Advanced HIV Disease. Science 1997, 277:112-116.

9. Yamashita TE, Phair JP, Muñoz A, Margolick JB, Detels R, et al: Immunologic and virologic response to highly active antiretroviral therapy in the Multicenter AIDS Cohort Study. AIDS 2001, 15:735-745.

10. DHHS: Guidelines for the use of Antiretroviral Agents in HIV-1 Infected Adults and Adolescent. 2008 [http://aidsinfo.nih.gov], date last accessed.

11. Celada F, Seiden PE: A computer model of cellular interaction in the immune system. Immunology Today 1992, 13(2):56-62.

12. Bernaschi M, Castiglione F: Design and implementation of an immune system simulator. Computers in Biology and Medicine 2001, 31(5):303-331

13. Farmer JD, Packard NH, Perelson AS: The immune system, adaptation and machine learning. Physica D 1986, 22:187-204.

14. Baldazzi V, Paci P, Bernaschi M, Castiglione F: Modeling lymphocytes homing and encounters in lymph nodes. BMC Bioinformatics 2009, 10:387.

15. Richards FJ: A flexible growth function for empirical use. J Exp Bot 1959, 10:290-300.

16. Fiebig EW, Wright DJ, Rawal BD, Garrett PE, Schumacher RT, Peddada L, Heldebrant C, Smith R, Conrad A, Kleinman SH, Busch MP: Dynamics of HIV viremia and antibody seroconversion in plasma donors: implications for diagnosis and staging of primary HIV infection. AIDS 2003, 17:1871-1879.

\section{Pre-publication history}

The pre-publication history for this paper can be accessed here: http://www.biomedcentral.com/1471-2334/11/56/prepub

doi:10.1186/1471-2334-11-56

Cite this article as: Paci et al.: Timely HAART initiation may pave the way for a better viral control. BMC Infectious Diseases 2011 11:56.

\section{Submit your next manuscript to BioMed Central and take full advantage of:}

- Convenient online submission

- Thorough peer review

- No space constraints or color figure charges

- Immediate publication on acceptance

- Inclusion in PubMed, CAS, Scopus and Google Scholar

- Research which is freely available for redistribution

Submit your manuscript at www.biomedcentral.com/submit
CioMed Central 